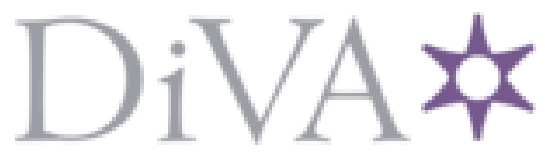

http://www.diva-portal.org

This is the published version of a paper published in Physical Review Letters.

Citation for the original published paper (version of record):

Khrennikov, K., Wenz, J., Buck, A., Xu, J., Heigoldt, M. et al. (2015)

Tunable All-Optical Quasimonochromatic Thomson X-Ray Sourcein the Nonlinear Regime.

Physical Review Letters, 114(19): 195003

https://doi.org/10.1103/PhysRevLett.114.195003

Access to the published version may require subscription.

N.B. When citing this work, cite the original published paper.

Permanent link to this version:

http://urn.kb.se/resolve?urn=urn:nbn:se:umu:diva-134416 


\title{
Tunable All-Optical Quasimonochromatic Thomson X-Ray Source in the Nonlinear Regime
}

\author{
K. Khrennikov, ${ }^{1,2}$ J. Wenz, ${ }^{1,2}$ A. Buck, ${ }^{2}$ J. Xu, ${ }^{2,3}$ M. Heigoldt, ${ }^{1,2}$ L. Veisz, ${ }^{2, *}$ and S. Karsch ${ }^{1,2, \dagger}$ \\ ${ }^{1}$ Ludwig-Maximilians-Universität München, Am Coulombwall 1, 85748 Garching, Germany \\ ${ }^{2}$ MPI für Quantenoptik, Hans-Kopfermann-Strasse 1, 85748 Garching, Germany \\ ${ }^{3}$ State Key Laboratory of High Field Laser Physics, Shanghai Institute of Optics and Fine Mechanics, \\ Chinese Academy of Sciences, P. O. Box 800-211, Shanghai 201800, China
}

(Received 20 August 2014; published 14 May 2015)

\begin{abstract}
We present an all-laser-driven, energy-tunable, and quasimonochromatic x-ray source based on Thomson scattering from laser-wakefield-accelerated electrons. One part of the laser beam was used to drive a few-fs bunch of quasimonoenergetic electrons, while the remainder was backscattered off the bunch at weakly relativistic intensity. When the electron energy was tuned from 17-50 MeV, narrow x-ray spectra peaking at 5-42 keV were recorded with high resolution, revealing nonlinear features. We present a large set of measurements showing the stability and practicality of our source.
\end{abstract}

DOI: 10.1103/PhysRevLett.114.195003

PACS numbers: 52.38.Ph, 52.25.Os, 52.50.Dg

Laser-wakefield-acceleration (LWFA) [1,2] has recently been shown to produce stable [3] quasimonoenergetic [4-6], energy tunable [7,8], MeV-GeV-scale [9,10] electron bunches with some tens of $\mathrm{pC}$ charge. Their short duration $[11,12]$, small transverse bunch size, and low divergence [13-15], typically of the order of a few fs, $\mu \mathrm{m}$, and mrad, respectively, lead to emittance figures rivaling that of the best conventional state-of-the-art linear accelerators.

These unique properties qualify them as a driver for compact $\mathrm{x}$-ray sources $[16,17]$ with high peak brilliance, which might close the gap in performance and price between large-scale synchrotrons and laboratory x-ray tubes, while offering inherent synchronization to the laser and electron beam.

Wiggling these low-emittance electron beams either in alternating magnetic field structures (undulator radiation $[18,19]$ ), strongly focusing plasma fields (betatron radiation [20,21]), or intense laser fields (Thomson-Compton scattering [22-25]) produces x-ray beams, in the latter two cases with extremely high peak brilliance for photon energies above $10 \mathrm{keV}$.

For comparison, the ELBE radio frequency (rf) linear accelerator team at Forschungszentrum Dresden-Rossendorf recently reported the generation of thirteen $12.3-\mathrm{keV}$ Thomson-scattering photons/shot in a $1.13 \mu$ sr solid angle from a $1.2 \mathrm{pC}$ electron beam at $22.5 \mathrm{MeV}$ with 4 ps duration [26]. Our LWFA-driven source produces 10 times more photons/solid angle/pC charge from a less than 3.5 times smaller spot, in a 1000 times shorter pulse, albeit with an approximately fourfold energy spread. From these numbers it becomes clear that LWFA-driven beams surpass current $\mathrm{rf}$ linac-based hard x-ray sources by more than 4 orders of magnitude in peak brilliance.

These unique properties hold great promise for extending the scope of applications in many research fields, from clinical phase-contrast imaging and material research to few-fs time-resolved x-ray emission spectroscopy and ultrafast pump-probe studies of structural dynamics.

Of the three LWFA-based x-ray generation schemes mentioned above, Thomson scattering offers the most promising route to well-controlled monochromatic $\mathrm{keV}$ to $\mathrm{MeV} \mathrm{x}$-ray beams due to the short wiggling period of the colliding laser field. Compared to previous work [24,25], we report the first direct measurement of quasimonochromatic LWFA-based Thomson spectra in the nonlinear regime without reliance on inverse spectral reconstruction algorithms. Apart from this we show that the shock-front injection scheme for LWFA appears to be perfectly suited for future use and further development of the source.

Neglecting higher harmonics, the energy of the emitted photons for the case of a near head-on collision of the laser pulse and a bunch of relativistic electrons is given by $[27,28]$

$$
\begin{aligned}
\hbar \omega_{\mathrm{x} \text {-ray }} & =\hbar \omega_{\text {Laser }} \frac{2\left(1+\beta \cos \theta_{I}\right) \gamma^{2}}{1+a_{0}^{2} / 2+\gamma^{2} \theta_{O}^{2}} \\
& \approx 1.55 \mathrm{eV} \frac{4 \gamma^{2}}{1+a_{0}^{2} / 2+\gamma^{2} \theta_{O}^{2}} .
\end{aligned}
$$

Here, $\omega_{\text {x-ray }}$ and $\omega_{\text {Laser }}$ are the frequencies of the scattered radiation and colliding pulse, $\gamma$ and $\beta$ are the relativistic quantities of the electrons. $a_{0}=e E_{\mathrm{Laser}} / m_{e} \omega_{\mathrm{Laser}} c$ is the normalized vector potential of the wiggling laser field, where $e, m_{e}, E_{\text {Laser }}, c$ are the electron charge, rest mass, laser electric field, and speed of light, respectively. $\theta_{I}$ and $\theta_{O}$ are the interaction and observation angles. The emitted radiation is monochromatic for collimated, monoenergetic electron and laser beams, $a_{0} \ll 1$, and an infinitely small observation area. 
For head-on collision and relativistic intensities of the colliding pulse the number of generated x-ray photons $N_{X}$ per shot is given by $[27,28]$

$$
N_{X}=\frac{\pi}{3} \alpha N_{0} N_{e} a_{0}^{2} \frac{1}{\langle n\rangle}\left[\frac{1-\beta}{1+\beta}+\frac{a_{0}^{2}}{4 \gamma^{2}} \frac{(1-\beta)}{(1+\beta)^{2}}\right]
$$

Here, $\alpha=e^{2} / \hbar c$ is the fine structure constant, $N_{0}$ and $N_{e}$ the number of laser periods and electrons, respectively, and $\langle n\rangle$ denotes the averaged harmonic number, which is close to unity in our weakly relativistic case.

The duration of the scattered x-ray pulse $\Delta T_{X}$ is given by the convolution of the electron bunch envelope with a twice Lorentz-contracted colliding pulse envelope. For Gaussian laser and electron pulses, one obtains

$$
\Delta T_{X} \approx \sqrt{\left(\Delta T_{\mathrm{el}}\right)^{2}+\left(\Delta T_{\text {Laser }} / 4 \gamma^{2}\right)^{2}}
$$

Here $\Delta T_{\text {el }}$ and $\Delta T_{\text {Laser }}$ are the FWHM electron bunch and laser pulse durations. For high electron energies $(\gamma \gg 1)$ the $\mathrm{x}$-ray pulse reflects the electron pulse duration of a few femtoseconds $[11,12]$.

The electron bunches for radiating the $\mathrm{x}$ rays were produced by LWFA in an energy-tunable, quasimonoenergetic fashion (see Fig. 3, left). When an intense laser pulse $\left(a_{0}>1\right)$ travels through an underdense plasma, it drives a plasma wave by repelling electrons from regions of high intensity by its ponderomotive force. The phase velocity of this wave equals the laser's group velocity, which is close to the speed of light. The wave constitutes a fast-moving longitudinal field structure that accelerates electrons once they are trapped [1,2]. In order to avoid large energy-spread beams due to the stochastic nature of self-trapping, a forced trapping scheme called shock-front injection [7,29] was employed. Placing a razor edge into the supersonic flow of a conical de Laval nozzle creates a shock front with a sharp density drop over the scale of the molecular mean free path $(1.3 \mu \mathrm{m}$ inferred from interferometric gas density measurements) in the laser propagation direction [29]. Upon crossing the drop the plasma wavelength instantaneously increases. This places the former first wave crest into the trough of the now elongated wave- a position with a strong accelerating field, trapping a large number of electrons at a defined position. They all experience the same acceleration distance and energy gain. The position of the shock in the gas target determines the acceleration length and final energy. If the plasma density is kept below the self-injection limit (by lowering the density such that without the shock front no electrons are observed), no further electrons are injected and the absolute energy spread stays small.

The experimental setup is depicted in Fig. 1, along with the details of the experiment. It produces electron beams with an energy peak tunable between 10 and $150 \mathrm{MeV}$, with a constant energy bandwidth of $5 \mathrm{MeV}$ FWHM and an average charge of $20 \mathrm{pC}$. For a limited energy range the

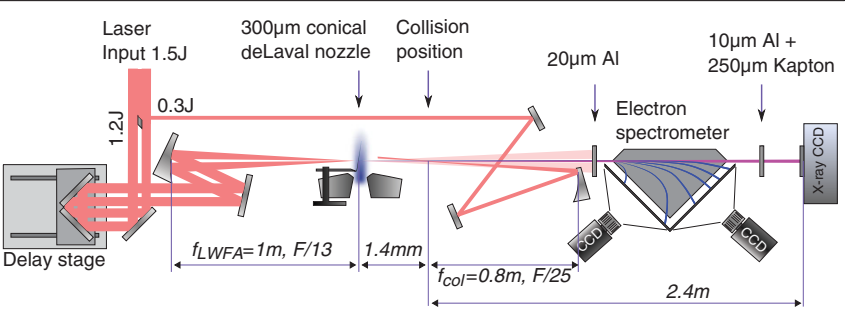

FIG. 1 (color). Experimental setup. $0.8 \mu \mathrm{m}$-wavelength, 28 fsduration pulses from the ATLAS-60 TW Ti:sapphire laser system at MPQ are split into a driver $(1.2 \mathrm{~J})$ and colliding beam $(0.3 \mathrm{~J})$. They are focused to $4.2 \times 10^{19} \mathrm{~W} / \mathrm{cm}^{2} \quad\left(a_{0}=4.4\right)$ and $1.8 \times 10^{18} \mathrm{~W} / \mathrm{cm}^{2}\left(a_{0}=0.9\right)$, respectively. The driver accelerates electrons from a plasma $\left(n_{e}=5 \times 10^{19} \mathrm{~cm}^{-3}\right)$ in a laserionized supersonic He gas flow from a $300 \mu \mathrm{m}$ conical de Laval nozzle. A razor blade creates a shock-front electron injector. The electron beam is analyzed on a scintillator screen behind a calibrated 1 T-dipole magnet spectrometer [30]. The colliding beam is focused $1.4 \mathrm{~mm}$ behind the electron injection point at a collision angle of $3.7^{\circ}$. X rays are detected on axis after $30-\mu \mathrm{m}$ aluminum and $250 \mu \mathrm{m}$ Kapton windows by either a scintillatorbased or a single-photon counting x-ray CCD camera.

beam divergence was measured during this campaign. It ranged from $17.5 \mathrm{mrad}$ at $27 \mathrm{MeV}$ to $12 \mathrm{mrad}$ at $45 \mathrm{MeV}$. This is comparable with the values reported in Ref. [7], where the electrons were produced under the same conditions as here.

The $300 \mathrm{~mJ}, 28 \mathrm{fs}$ colliding pulse is focused to a spot size of $25 \mu \mathrm{m} \mathrm{FWHM}$ and a peak $a_{0}=0.9$ to a point $1.4 \mathrm{~mm}$ behind the shock front on the drive laser axis. This $a_{0}$ allows us to study Thomson scattering in the weakly nonlinear regime $[27,28]$. This collision point was chosen to keep the colliding beam from disturbing the electron acceleration and to keep the interaction point in vacuum (cf. nozzle diameter $0.3 \mathrm{~mm}$ ). The spot size roughly corresponds to the electron beam diameter at this position (see Fig 1), as deduced from a few- $\mu$ m size inside the plasma [8] and the measured electron divergence.

Shadowgraphy was used for temporal alignment of the colliding pulse with the electron bunch. A small part of the laser beam, timed by a delay stage, acts as a transverse probe of the interaction. The ionization fronts of the drive and colliding pulse leave an imprint in the probe wave front and lead to intensity modulations reflecting the electron density gradients. Scanning the delay of the probe beam allows us to track the progression of the ionization fronts. By observing both the drive and colliding beam separately, the collision point relative to the electron injection and acceleration regions can be precisely determined.

For transverse alignment of both beams a top- and a side-view imaging system was used which monitors the Thomson side-scattered light from the background plasma electrons, allowing us to overlap the beams at the intended collision position. More details on the alignment procedure can be found in the Supplemental Material [31]. 
(a) $30 \mathrm{MeV}$ electrons

$15 \mathrm{keV}$ photons

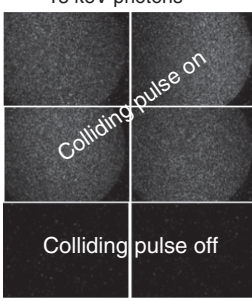

(b) $50 \mathrm{MeV}$ electrons

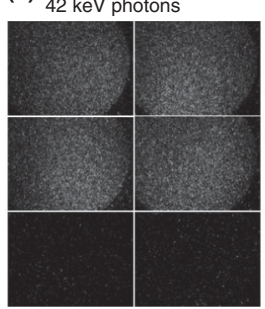

(c) $70 \mathrm{MeV}$ electrons

(c) $83 \mathrm{keV}$ photons

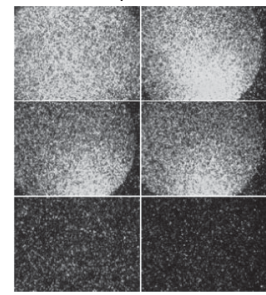

FIG. 2. Images of the x-ray beam from the scintillatorintensified camera. Series (a), (b), and (c) show raw data at an electron energy of 30,50 , and $70 \mathrm{MeV}$, corresponding to x-ray photon energies of 15,42 , and $83 \mathrm{keV}$, respectively. The four top images show the CCD signal with the colliding beam on, the two lower images in each section were taken with a blocked colliding beam. The gain of the MCP was doubled for image (c) to compensate for the lower scintillator efficiency at this energy. Because of enhanced beaming, the brightness of series (a) and (b) seems to be equal in spite of an eightfold reduction in the scintillator efficiency at the higher energy in (b).

The x-ray beam is emitted from a source reflecting the size of the electron bunch at the collision point within an angle given by the convolution of the electron beam divergence and a $1 /\left(\gamma N_{0}^{1 / 2}\right)$ radius cone [27,28] due to relativistic beaming, where $N_{0} \approx 10$ denotes the number of laser oscillations.

Figure 2 shows the $\mathrm{x}$ rays from 30,50 , and $70 \mathrm{MeV}$ electrons, peaked at 15,42 , and $83 \mathrm{keV}$, respectively. A clear on-off behavior is evident, indicating that the $\mathrm{x}$ rays come from the electron-laser interaction and not from betatron oscillations during the acceleration process or bremsstrahlung of electrons hitting the chamber walls. A 2-in. diameter Proxitronic scintillator-based intensified $\mathrm{x}$-ray camera with variable gain was used for detection. It offers spatial resolution and high sensitivity between 2.5 and $100 \mathrm{keV}$, but no absolute single-photon energy information.

In order to obtain single-shot x-ray spectra, a backilluminated x-ray CCD camera (Andor DO432 BN-DD) with $1250 \times 115222.5 \mu \mathrm{m}$-size pixels operating in singlephoton-counting mode was used. This technique exploits the fact that camera readout counts are directly proportional to the absorbed $\mathrm{x}$-ray photon energy in each pixel. In practice, the energy of a single photon is deposited in a cluster of neighboring pixels. In a post-processing step the content of all these pixels is added up to yield the photon energy, provided the clusters represent a single photon. This sets a limit on the number of detectable photons per shot, which, in our case, according to Poisson statistics, is 40000 if the double-hit probability is kept below $2 \%$. Practically, one sets this limit by choosing an appropriate source-detector distance. The histograms of the postprocessed images corrected by the camera sensitivity and material absorption (for $30 \mu \mathrm{m}$ aluminum, $250 \mu \mathrm{m}$ Kapton, and $3 \mathrm{~cm}$ air) yield the absolute $\mathrm{x}$-ray spectrum of the source, averaged over the $0.13 \mathrm{msr}$ solid angle of the $28.1 \times 25.9 \mathrm{~mm}^{2}$ chip at a $2.4 \mathrm{~m}$ distance. As the CCD

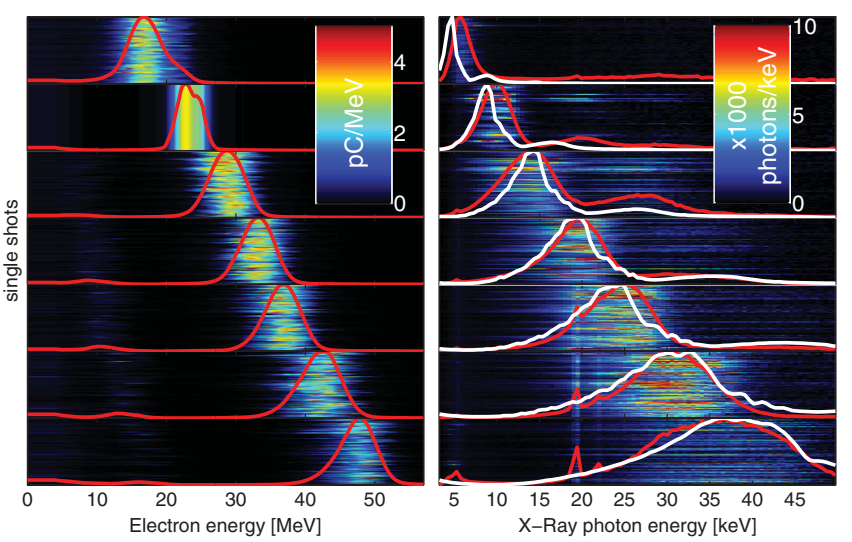

FIG. 3 (color). Electron (left) and corresponding x-ray photon spectra (right). Each horizontal trace is a single laser shot. Shown are the best $50 \%$ of shots by photon number in each run. Different horizontal sections correspond to different razor-blade positions and electron beam energy. X-ray spectra are corrected for filter and vacuum window transmission (see Fig. 1) and CCD sensitivity. Run-averaged spectra are shown in red, white lines show the expected x-ray spectrum for each averaged electron spectrum. The simulation was performed with SPECTRA 9.0 [42], assuming an equivalent undulator model with a Gaussian field envelope and a peak undulator parameter of $K=a_{0}=0.9$.

quantum efficiency almost vanishes above $\sim 40 \mathrm{keV}$, only the spectra of runs with lower photon energy are presented in Fig. 3 along with their respective electron spectra. Photon numbers are given per solid angle, since the beam divergence is considerably larger than the detector for lower energies, making an estimate of total photon yield difficult.

Normalized, run-averaged electron and photon spectra are plotted as red lineouts on the left and right panel of Fig. 3, respectively. Their shape seems to be in good agreement with the expectation (white curve) from the respective electron spectra and a collision angle of $3.7^{\circ}$. This angle was chosen to prevent damage to the laser system by back-propagating light. The simulation was performed using the SPECTRA 9.0 code [42]. Although this code was designed to simulate a purely magnetic interaction of relativistic electrons with an undulator, it delivers accurate results for our interaction regime of relatively weak laser and highly relativistic electrons. This accuracy was verified by comparing its results for single electron interaction with the results of the RTDX [43] code.

In the experiment, the shock-front injection produced quasimonochromatic beams in $>90 \%$ of all laser shots, with their peak energy stable within $3 \% \mathrm{rms}$ and a relative shot-to-shot charge fluctuation of $30 \% \mathrm{rms}$. The beam collision and $\mathrm{x}$-ray production was successful in $>76 \%$ of all laser shots, yielding $\mathrm{x}$-ray spectral peaks stable to within $10 \% \mathrm{rms}$ in energy and $75 \% \mathrm{rms}$ in photon number. Apart from possible electron bunch footprint variations, a prominent reason for the shot-to-shot variations in photon number is the pointing instability of the laser caused by building vibrations, which in the chosen geometry did not 


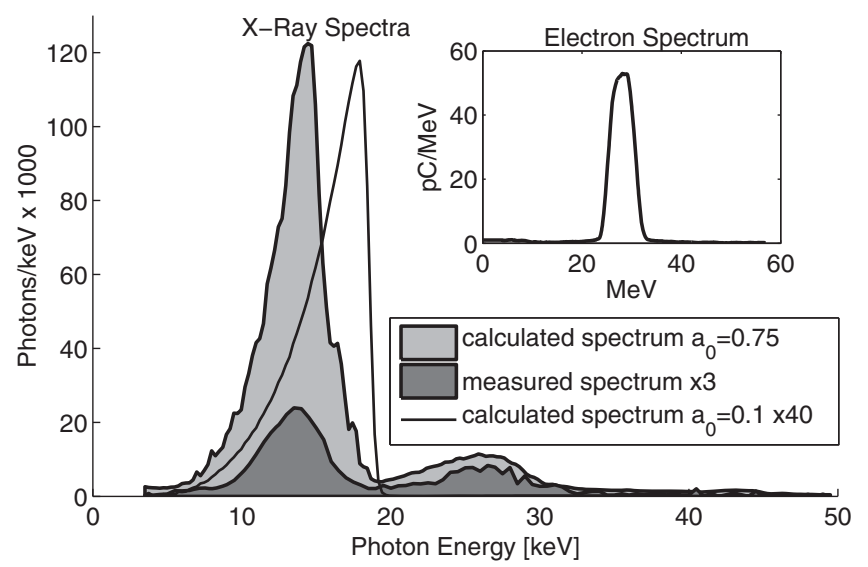

FIG. 4. Single representative x-ray spectrum (multiplied by 3), corresponding electron spectrum (inset), and x-ray spectrum calculated by SPECTRA 9.0 [42] for an interaction at $a_{0}=0.75$.

cancel out for the two beams. We are confident that this stability can be improved with an optimized setup in the future.

A representative single-shot spectrum together with its absolute theoretical expectation is shown in Fig 4. Note the presence of the 2nd order harmonic emission, which is clearly visible here due to the finite beam divergence and detection angle, while the third order is beyond the CCD sensitivity.

In Fig. 3, the photon energy scales approximately quadratic with the electron energy as expected from Eq. (1). This observation is elaborated in Fig. 5, where the positions of the electron and x-ray spectral peaks and their corresponding rms spectral widths (in gray) are

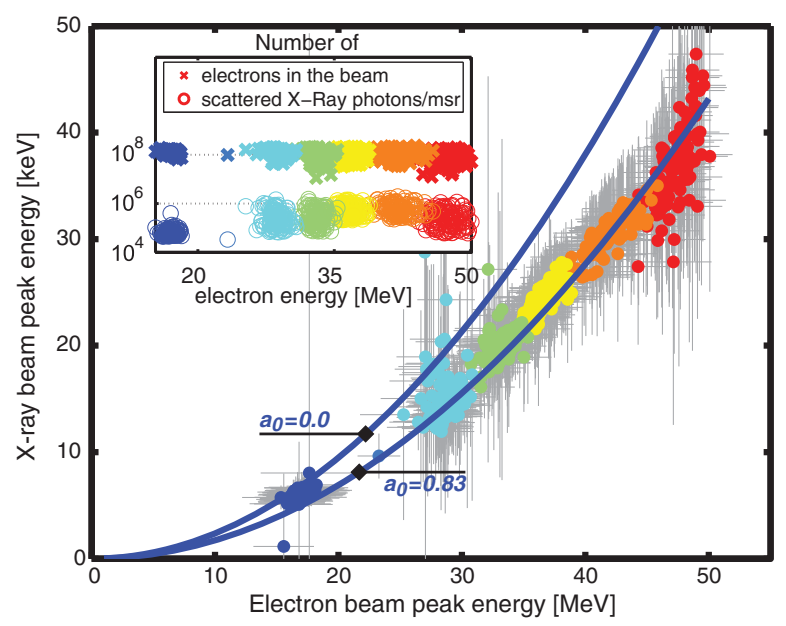

FIG. 5 (color). Spectral peaks positions, determined by a Gaussian fit to the measured spectra, whose rms width defines the error bars. Each color stands for a different shock position, indicating the reproducibility of each setting. The blue line labeled $a_{0}=0.83$ is a quadratic best fit [according to Eq. (1)] to the measured x-ray scaling, while the one for $a_{0}=0$ serves as a comparison. The inset shows the measured electron and $\mathrm{x}$-ray photon number/msr for each shot. plotted for each shot in comparison with the expected scalings for different electric field strengths.

The best fit to the experimental data, indicating a peak $a_{0}$ of 0.83 is in good agreement with $a_{0}=0.9$ inferred from the focal spot, laser duration, and energy measurements. This proves that the $a_{0}^{2}$ factor in Eq. (1) cannot be neglected, and confirms the onset of the nonlinear scattering regime. Therefore, the x-ray spectral widths (vertical error bars) have to be attributed not only to the energy bandwidth of the electron bunch and laser pulse, but also to the Gaussian temporal intensity profile of the collision pulse. The inset shows the total electron numbers and $\mathrm{x}$-ray photon numbers/msr. Since the $\mathrm{x}$-ray divergence is larger than the CCD chip, it is not possible to extract $\mathrm{x}$-ray divergence figures from the measurement for computing total photon numbers.

With an estimated electron bunch duration of $5 \mathrm{fs}$, as measured under similar experimental conditions [11,12] and taking into account the duration of the colliding pulse, Eq. (3) yields an $\mathrm{x}$-ray pulse duration of approximately $5 \mathrm{fs}$. We estimate the upper limit for the brilliance of the x-ray source based on the detected x-ray photon numbers. Since the electrons come from a $2 \mu \mathrm{m}$ FWHM source [12] with the directly measured divergence of $20 \ldots 12 \mathrm{mrad}$ FWHM (decreasing with electron energy) this translates to $0.2 \ldots 7.4 \times 10^{20}[$ (photons $) /\left(\mathrm{s} \mathrm{mm}^{2} \mathrm{mrad}^{2} 0.1 \%\right.$ bandwidth $\left.)\right]$ for the case of the interaction at the electron beam waist. The range is due to the energy dependence of the electron divergence affecting the brilliance, where $2 \times 10^{19}$ corresponds to $6 \mathrm{keV}$ photons and $7.4 \times 10^{20}$ to $42 \mathrm{keV}$.

In the experiment, the collision point was deliberately shifted $1.4 \mathrm{~mm}$ downstream into the vacuum (see Fig. 1) to exclude any plasma disturbance by the colliding pulse. At this position the electron bunch has expanded to a diameter of $30 \ldots 13 \mu \mathrm{m}$ FWHM (cf. energy-dependent divergence), which leads to a reduced $\mathrm{x}$-ray brilliance of $0.2 \ldots 15 \times 10^{18}[$ (photons $) /\left(\mathrm{smm}^{2} \mathrm{mrad}^{2} 0.1 \%\right.$ bandwidth $\left.)\right]$. We expect that in future experiments this vacuum propagation can be significantly reduced to approach the maximum brilliance figures.

As detailed in the introduction and the Supplemental Material [31], our source compares well with other x-ray sources of comparable photon energy. At 9 orders of magnitude above bremsstrahlung, 4-5 orders above compact storage ring and enhancement cavity sources, and 3-4 orders above rf-linac and Thomson sources, our peak brilliance is only bettered by LWFA/betatron sources and 3rd generation rf storage rings (ESRF, PETRA III) (see Supplemental Material [31], and references therein). While the former offer no tunability and a broad, synchrotronlike spectrum, the latter are large-scale facilities with limited access. We expect that after an ongoing laser upgrade, an expected 5-10x increase in bunch charge [7] and scattering laser fluence will yield a $50 \times-100 \times$ improvement over the current state. 
With better detectors for higher energy photons, the usable energy range of our source can be easily scaled. The shock-front acceleration scheme routinely delivers quasimonoenergetic electron bunches with energies up to $150 \mathrm{MeV}$ [7], extending the photon energy to $400 \mathrm{keV}$.

The onset of the nonlinear Thomson backscattering regime has been observed, to our knowledge for the first time, as a direct manifestation in the $\mathrm{x}$-ray spectrum.

Further developments will center on optimizing the colliding pulse by tailoring [44] it to a small collision radius, high colliding flux, but low intensity in order to enhance the scattered $\mathrm{x}$-ray flux while keeping its spectrum narrow.

By combining this concept with future high-repetition rate laser systems based on OPCPA as the electron driver and using a part of their high-repetition rate picosecond pump lasers $[45,46]$ as a colliding pulse, a resulting Thomson source could serve a variety of imaging applications in research, medicine, and industry due to its compactness, tunability, and unmatched short pulse duration.

This work was supported by DFG through the Cluster of Excellence MAP (EXC 158) and TR-18 funding schemes, Euratom research and training programme 2014-2018 under Grant agreement No. 633053 within the framework of the EUROfusion Consortium, and the Max- PlanckSociety.

*laszlo.veisz@mpq.mpg.de †stefan.karsch@mpq.mpg.de

[1] T. Tajima and J. M. Dawson, Phys. Rev. Lett. 43, 267 (1979).

[2] E. Esarey, C. B. Schroeder, and W. P. Leemans, Rev. Mod. Phys. 81, 1229 (2009).

[3] J. Osterhoff et al., Phys. Rev. Lett. 101, 085002 (2008).

[4] C. G. R. Geddes, Cs. Toth, J. van Tilborg, E. Esarey, C. B. Schroeder, D. Bruhwiler, C. Nieter, J. Cary, and W. P. Leemans, Nature (London) 431, 538 (2004).

[5] J. Faure, Y. Glinec, A. Pukhov, S. Kiselev, S. Gordienko, E. Lefebvre, J.-P. Rousseau, F. Burgy, and V. Malka, Nature (London) 431, 541 (2004).

[6] S. P. D. Mangles et al., Nature (London) 431, 535 (2004).

[7] A. Buck et al., Phys. Rev. Lett. 110, 185006 (2013).

[8] A. J. Gonsalves et al., Nat. Phys. 7, 862 (2011).

[9] W. P. Leemans, B. Nagler, A. J. Gonsalves, Cs. Toth, K. Nakamura, C. G. R. Geddes, E. Esarey, C. B. Schroeder, and S. M. Hooker, Nat. Phys., 2, 696 (2006).

[10] S. Kneip et al., Phys. Rev. Lett. 103, 035002 (2009).

[11] O. Lundh et al., Nat. Phys. 7, 219 (2011).

[12] A. Buck, M. Nicolai, K. Schmid, C. M. S. Sears, A. Sävert, J. Mikhailova, F. Krausz, M. Kaluza, and L. Veisz, Nat. Phys. 7, 543 (2011).

[13] R. Weingartner et al., Phys. Rev. ST Accel. Beams 15, 111302 (2012).

[14] E. Brunetti et al., Phys. Rev. Lett. 105, 215007 (2010).

[15] C. M. S. Sears, A. Buck, K. Schmid, J. Mikhailova, F. Krausz, and L. Veisz, Phys. Rev. ST Accel. Beams 13, 092803 (2010).
[16] S. Corde, K. Ta Phuoc, G. Lambert, R. Fitour, V. Malka, A. Rousse, A. Beck, and E. Lefebvre, Rev. Mod. Phys. 85, 1 (2013).

[17] T Pfeifer, C Spielmann, and G Gerber, Rep. Prog. Phys. 69, 443 (2006).

[18] H. P. Schlenvoigt et al., Nat. Phys. 4, 130 (2008).

[19] M. Fuchs et al., Nat. Phys. 5, 826 (2009).

[20] A. Rousse et al., Phys. Rev. Lett. 93, 135005 (2004).

[21] S. Kneip et al., Nat. Phys. 6, 980 (2010).

[22] H. Schwoerer, B. Liesfeld, H. P. Schlenvoigt, K. U. Amthor, and R. Sauerbrey, Phys. Rev. Lett. 96, 014802 (2006).

[23] K. Ta Phuoc, S. Corde, C. Thaury, V. Malka, A. Tafzi, J. P. Goddet, R. C. Shah, S. Sebban, and A. Rousse, Nat. Photonics 6, 308 (2012).

[24] S. Chen et al., Phys. Rev. Lett. 110, 155003 (2013).

[25] N. D. Powers, I. Ghebregziabher, G. Golovin, C. Liu, S. Chen, S. Banerjee, J. Zhang, and D. P. Umstadter, Nat. Photonics 8, 28 (2013).

[26] A. Jochmann et al., Phys. Rev. Lett. 111, 114803 (2013).

[27] E. Esarey and P. Sprangle, Phys. Rev. E 48, 3003 (1993).

[28] K. Ride, E. Esarey, and M. Baine, Phys. Rev. E 52, 5425 (1995).

[29] K. Schmid, A. Buck, C. M. S. Sears, J. M. Mikhailova, R. Tautz, D. Herrmann, M. Geissler, F. Krausz, and L. Veisz, Phys. Rev. ST Accel. Beams 13, 091301 (2010).

[30] C. M. S. Sears, S. B. Cuevas, U. Schramm, K. Schmid, A. Buck, D. Habs, F. Krausz, and L. Veisz, Rev. Sci. Instrum. 81, 073304 (2010).

[31] See Supplemental Material at http://link.aps.org/supplemental/ 10.1103/PhysRevLett.114.195003 for a detailed description of the collision alignment procedure and the overview of the recently achieved peak brilliances for different $\mathrm{x}$-ray generation mechanisms, which includes Refs. [32-41].

[32] http://www.rigaku.com/products/protein/fre

[33] http://www.esrf.eu/Accelerators/Performance/Brilliance

[34] http://petra3.desy.de/index_ger.html

[35] M. Uesaka et al., Nucl. Instrum. Methods Phys. Res., Sect. A 455, 90 (2000).

[36] Y. Du, L. Yan, J. Hua, Q. Du, Z. Zhang, R. Li, H. Qian, W. Huang, H. Chen, and C. Tang, Rev. Sci. Instrum. 84, 053301 (2013).

[37] F. Albert et al., Phys. Rev. ST Accel. Beams 13, 070704 (2010).

[38] G. Sarri et al., Phys. Rev. Lett. 113, 224801 (2014).

[39] R. J. Loewen, Ph.D.thesis, Stanford University, 2003.

[40] A. Zelinsky et al., in Proceedings of the 9th European Particle Accelerator Conference, 2004, Published as SLACPUB-11118, http://www.slac.stanford.edu/cgi-wrap/getdoc/ slac-pub-11118.pdf.

[41] K. Dupraz et al., Phys. Rev. ST Accel. Beams 17, 033501 (2014).

[42] T. Tanaka and H. Kitamura, J. Synchrotron Radiat. 8, 1221 (2001).

[43] A. G. R. Thomas, Phys. Rev. ST Accel. Beams 13, 020702 (2010).

[44] A. D. Debus et al., Appl. Phys. B 100, 61 (2010).

[45] J. Tümmler, R. Jung, H. Stiel, P. V. Nickles, and W. Sandner, Opt. Lett. 34, 1378 (2009).

[46] G. Mourou, B. Brocklesby, T. Tajima, and J. Limpert, Nat. Photonics 7, 258 (2013). 